\title{
SPECTRAL ANALYSIS FOR DIFFERENTIAL OPERATORS WITH SINGULARITIES
}

VJACHESLAV ANATOLJEVICH YURKO

Received 15 November 2001

Nonselfadjoint boundary value problems for second-order differential equations on a finite interval with nonintegrable singularities inside the interval are considered under additional sewing conditions for solutions at the singular point. We study properties of the spectrum, prove the completeness of eigen- and associated functions, and investigate the inverse problem of recovering the boundary value problem from its spectral characteristics.

\section{Introduction}

We consider the differential equation

$$
-y^{\prime \prime}+\left(\frac{\nu_{0}}{(x-a)^{2}}+q(x)\right) y=\lambda y, \quad 0<x<T, a \in(0, T)
$$

with nonintegrable singularity inside the interval. Here, $q(x)$ is a complex-valued function, and $\nu_{0}$ is a complex number. Let $\nu_{0}=v^{2}-1 / 4$, and for definiteness, let $\operatorname{Re} v>0$, $v \notin \mathbb{N}$. We will assume that $q(x)|x-a|^{\min (0,1-2 \operatorname{Re} v)} \in L(0, T)$.

This paper deals with the boundary value problem $L$ for the differential equation (1.1) with the boundary conditions

$$
U(y):=y^{\prime}(0)-h y(0)=0, \quad V(y):=y^{\prime}(T)+H y(T)=0,
$$

and with additional "sewing condition" for solutions at the singular point $x=a$. We consider in some sense general sewing conditions defined by a transition matrix

$$
A=\left[a_{j k}\right]_{j, k=1,2}
$$

which connects solutions of (1.1) near the singular point (see Section 3 for details). In the particular case of the absence of the singularity $(\nu=1 / 2)$, these sewing conditions 
correspond to the following jump conditions

$$
\left[\begin{array}{l}
y \\
y^{\prime}
\end{array}\right](a+0)=A\left[\begin{array}{l}
y \\
y^{\prime}
\end{array}\right](a-0) .
$$

Problem (1.1), (1.2), and (1.4) without singularities $(\nu=1 / 2)$, was studied in $[10,11,26]$ and other works. In another particular case when $A=E$ ( $E$ is the identity matrix) and $\nu_{0}$ is an arbitrary complex number with $\operatorname{Re} v>0, v \notin \mathbb{N}$, the sewing conditions considered in this paper correspond (in the case when $q(x)$ is a regular function) to sewing solutions by the analytic continuation in the upper half-plane $\operatorname{Im} x>0$. This particular case was studied in $[24,25]$.

Differential equations with singularities inside the interval play an important role in various areas of mathematics as well as in its applications. Moreover, a wide class of differential equations with turning points can be reduced to equations with singularities. For example, such problems appear in electronics for constructing parameters of heterogeneous electronic lines with desirable technical characteristics $[7,15,18]$. After reduction of the corresponding mathematical model, we come to the inverse spectral problem for the boundary value problem $L$ where $q(x)$ must be constructed from the given spectral information which describes desirable amplitude and phase characteristics. Boundary value problems with discontinuities in an interior point appear in geophysical models for oscillations of the Earth $[2,13]$. Furthermore, direct and inverse spectral problems for equations with singularities and turning points are used for studying the blowup behavior of solutions for some nonlinear integrable evolution equations in mathematical physics (see, e.g., [5]). Other classes of boundary value problems with discontinuities were considered in $[1,6]$ and other works. We also note that in different problems of natural sciences, we face different kind of sewing conditions defined by different matrices A. This makes actual to study the boundary value problem $L$ with an arbitrary sewing condition.

In this paper, we study direct and inverse problems of spectral analysis for the boundary value problem $L$ defined by (1.1), (1.2) and a transition matrix $A$. Properties of the spectrum are obtained, the completeness of eigen- and associated functions (e.a.f.) is proved, and the inverse problem of recovering $L$ form its spectral characteristics is investigated.

For the classical Sturm-Liouville operators (when $v=1 / 2$ and $A=E$ ), direct and inverse problems of spectral analysis have been studied fairly completely (see $[9,14,16$, $17,19]$ and the references therein). The case when a singular point lies at the end of the interval $(a=0)$ was investigated in $[4,8,20,21,22,23,28]$ and in other works. The presence of singularity inside the interval produces essential qualitative modifications in the investigation of direct and inverse problems. For studying the boundary value problem $L$, an important role is played by special fundamental systems of solutions with necessary analytic, asymptotic, and structural properties which gives us an opportunity to investigate the behavior of the corresponding Stokes multipliers and to study the characteristic function and the so-called Weyl function. These fundamental systems of solutions are constructed in Section 2. In Section 3, properties of the spectrum and the characteristic function are studied. We introduce the so-called regularity condition for sewing. 
Under this condition, the completeness of e.a.f. is proved in Section 4. We also provide a counterexample showing the importance of the regularity condition for studying the boundary value problem $L$. In Section 5, the inverse spectral problem is considered for the boundary value problem $L$.

\section{Fundamental systems of solutions}

2.1. Let $\lambda=\rho^{2}$. Consider the functions

$$
C_{j}(x, \lambda)=(x-a)^{\mu_{j}} \sum_{k=0}^{\infty} c_{j k}(\rho(x-a))^{2 k}, \quad j=1,2,
$$

where

$$
\begin{gathered}
\mu_{j}=(-1)^{j} \nu+\frac{1}{2}, \quad c_{10} c_{20}=(2 \nu)^{-1}, \\
c_{j k}=(-1)^{k} c_{j 0}\left(\prod_{s=1}^{k}\left(\left(2 s+\mu_{j}\right)\left(2 s+\mu_{j}-1\right)-\nu_{0}\right)\right)^{-1} .
\end{gathered}
$$

Here and in the sequel, $z^{\mu}=\exp (\mu(\ln |z|+i \arg z)), \arg z \in(-\pi, \pi]$. For $x>a$ and $x<a$, the functions $C_{j}(x, \lambda)$ are the solutions of the equation

$$
-y^{\prime \prime}+\frac{\nu_{0}}{(x-a)^{2}} y=\lambda y
$$

Let $s_{j}(x, \lambda), j=1,2$, be solutions of the following integral equations for $x>a$ and $x<a$ :

$$
s_{j}(x, \lambda)=C_{j}(x, \lambda)+\int_{a}^{x} g(x, t, \lambda) q(t) s_{j}(t, \lambda) d t,
$$

where $g(x, t, \lambda)=C_{1}(t, \lambda) C_{2}(x, \lambda)-C_{1}(x, \lambda) C_{2}(t, \lambda)$. The functions $s_{j}(x, \lambda)$ are entire in $\lambda$ of order $1 / 2$, and form a fundamental system of solutions of (1.1). At that,

$$
\begin{gathered}
\operatorname{det}\left[s_{j}^{(m-1)}(x, \lambda)\right]_{j, m=\overline{1,2}} \equiv 1 \\
\left|s_{j}^{(m)}(x, \lambda)\right| \leq C\left|(x-a)^{\mu_{j}-m}\right|, \quad\left|s_{j}(x, \lambda)-C_{j}(x, \lambda)\right| \leq C\left|(x-a)^{2 v+\mu_{j}}\right|, \quad|\rho(x-a)| \leq 1 .
\end{gathered}
$$

Here and below, one and the same symbol $C$ denotes various positive constants in the estimates. We will call $s_{j}(x, \lambda)$ the Bessel-type solutions for (1.1).

Let $s_{j,-}(x, \lambda), x>a$, be the Bessel-type solutions for the equation

$$
-y_{-}^{\prime \prime}(x)+\left(\frac{v_{0}}{(x-a)^{2}}+q(2 a-x)\right) y_{-}(x)=\lambda y_{-}(x), \quad x>a .
$$

Then, the functions $s_{j}^{R}(x, \lambda):=s_{j,-}(2 a-x, \lambda), x<a$, are solutions of $(1.1)$. It is easily seen that

$$
s_{j}^{R}(x, \lambda)=\exp \left(-i \pi \mu_{j}\right) s_{j}(x, \lambda), \quad x<a .
$$




\section{Spectral analysis for differential operators with singularities}

Denote $S_{k_{0}}=\left\{\rho: \arg \rho \in\left(k_{0} \pi / 2,\left(k_{0}+1\right) \pi / 2\right)\right\}, k_{0}=\overline{-2,1}$. In each sector $S_{k_{0}}$, the roots $R_{k}, k=1,2$ of the equation $R^{2}+1=0$ can be numbered in such a way that $\operatorname{Re}\left(\rho R_{1}\right)<$ $\operatorname{Re}\left(\rho R_{2}\right), \rho \in S_{k_{0}}$. Clearly, $R_{k}=(-1)^{k-1} i$ for $S_{0}$ and $S_{1}$, and $R_{k}=(-1)^{k} i$ for $S_{-1}$ and $S_{-2}$. For definiteness, let $\operatorname{Re} \rho \geq 0$, that is, $\rho \in \overline{S_{0} \cup S_{-1}}$. In [21], a special fundamental system of solutions $\left\{y_{k}(x, \rho)\right\}_{k=1,2}, x>a, \rho \in S_{k_{0}}$, of the differential equation (1.1) in each sector $S_{k_{0}}$ is constructed having the following properties:

(1) for each $x \in(a, T]$, the functions $y_{k}^{(m)}(x, \rho), m=0,1$, are regular with respect to $\rho$ for $\rho \in S_{k_{0}},|\rho| \geq \rho_{*}$, are continuous for $\rho \in \bar{S}_{k_{0}},|\rho| \geq \rho_{*}$, and

$$
\begin{aligned}
& \left|y_{k}^{(m)}(x, \rho)\left(\rho R_{k}\right)^{-m} \exp \left(-\rho R_{k}(x-a)\right)-1\right| \\
& \quad \leq \frac{C}{|\rho(x-a)|}, \quad x \in(a, T], \rho \in \bar{S}_{k_{0}},|\rho(x-a)| \geq 1 ;
\end{aligned}
$$

(2) the relation

$$
y_{k}(x, \rho)=\sum_{j=1}^{2} b_{k j}^{+}(\rho) s_{j}(x, \lambda), \quad x>a
$$

holds, where

$$
b_{k j}^{+}(\rho)=b_{k j} \rho^{\mu_{j}}[1], \quad|\rho| \longrightarrow \infty,
$$

where [1] $:=1+O\left(\rho^{-1}\right)$, and the constants $b_{k j}$ depend on the sector. We will call $y_{k}(x, \rho)$ the Birkhoff-type solutions for (1.1).

Let $y_{k,-}(x, \rho), x>a$, be the Birkhoff-type solutions for (2.7). Then, the functions $y_{k}(x, \rho):=y_{k,-}(2 a-x, \rho), x<a$, are solutions of (1.1). Then, symmetrically to (2.10),

$$
y_{k}(x, \rho)=\sum_{j=1}^{2} b_{k j}^{-}(\rho) s_{j}^{R}(x, \lambda), \quad x<a,
$$

where

$$
b_{k j}^{-}(\rho)=b_{k j} \rho^{\mu_{j}}[1], \quad|\rho| \longrightarrow \infty
$$

with the same constants $b_{k j}$ as in (2.11). By virtue of (2.8) and (2.12),

$$
y_{k}(x, \rho)=\sum_{j=1}^{2} B_{k j}^{-}(\rho) s_{j}(x, \lambda), \quad x<a,
$$

where

$$
B_{k j}^{-}(\rho)=b_{k j}^{-}(\rho) \exp \left(-i \pi \mu_{j}\right)
$$


It follows from (2.10) and (2.14) that

$$
\begin{array}{ll}
s_{j}(x, \lambda)=\sum_{k=1}^{2} d_{j k}^{+}(\rho) y_{k}(x, \rho), & x>a, \\
s_{j}(x, \lambda)=\sum_{k=1}^{2} D_{j k}^{-}(\rho) y_{k}(x, \rho), & x<a,
\end{array}
$$

where

$$
D_{j k}^{-}(\rho)=d_{j k}^{-}(\rho) \exp \left(i \pi \mu_{j}\right),
$$

and $\left[d_{j k}^{ \pm}(\rho)\right]_{j, k=1,2}=\left(\left[b_{k j}^{ \pm}(\rho)\right]_{k, j=1,2}\right)^{-1}$. Using (2.11) and (2.13), we infer

$$
d_{j k}^{ \pm}(\rho)=d_{j k} \rho^{-\mu_{j}}[1], \quad|\rho| \longrightarrow \infty,
$$

where $\left[d_{j k}\right]_{j, k=1,2}=\left(\left[b_{k j}\right]_{k, j=1,2}\right)^{-1}$. We rewrite (2.9) as follows:

$$
y_{k}^{(m)}(x, \rho)=\left(\rho R_{k}\right)^{m} \exp \left(\rho R_{k}(x-a)\right)[1]_{a}, \quad x \in(a, T], \rho \in \bar{S}_{k_{0}},|\rho(x-a)| \geq 1,
$$

where $[1]_{a}=1+O(1 /|\rho(x-a)|),|\rho(x-a)| \geq 1$ (i.e. $f(x, \rho)=[1]_{a}$ means $|f(x, \rho)-1| \leq$ $C /|\rho(x-a)|,|\rho(x-a) \geq 1|)$. Similarly,

$$
y_{k}^{(m)}(x, \rho)=\left(-\rho R_{k}\right)^{m} \exp \left(\rho R_{k}(a-x)\right)[1]_{a}, \quad x \in[0, a), \rho \in \bar{S}_{k_{0}},|\rho(x-a)| \geq 1 .
$$

In particular, (2.19) and (2.20) yield $\operatorname{det}\left[y_{k}^{(m-1)}(x, \rho)\right]_{k, m=1,2}=\mp 2 i \rho[1], x \in J_{ \pm}$, where $J_{-}:=[0, a), J_{+}:=(a, T]$.

2.2. Let a matrix

$$
A=\left[\begin{array}{ll}
a_{11} & a_{12} \\
a_{21} & a_{22}
\end{array}\right], \quad \operatorname{det} A \neq 0
$$

be given, where $a_{k j}$ are complex numbers. We introduce the functions $\left\{\sigma_{j}(x, \lambda)\right\}_{j=1,2}$, $x \in[0, a) \cup(a, T]$, by the formula

$$
\sigma_{j}(x, \lambda)= \begin{cases}s_{j}(x, \lambda), & x<a, \\ \sum_{k=1}^{2} a_{k j} s_{k}(x, \lambda), & x>a .\end{cases}
$$

The functions $\sigma_{j}(x, \lambda)$ satisfy the differential equation (1.1) for $x<a$ and $x>a$, and according to (2.5),

$$
\operatorname{det}\left[\sigma_{j}^{(m-1)}(x, \lambda)\right]_{j, m=1,2} \equiv \begin{cases}1, & x<a \\ \operatorname{det} A, & x>a .\end{cases}
$$




\section{Spectral analysis for differential operators with singularities}

The fundamental system of solutions $\left\{\sigma_{j}(x, \lambda)\right\}$ will be used for sewing together solutions at the singular point $x=a$.

Using (2.10), (2.14), (2.16), and (2.22) we get

$$
y_{k}(x, \rho)=\sum_{j=1}^{2} B_{k j}^{ \pm}(\rho) \sigma_{j}(x, \lambda), \quad \sigma_{j}(x, \lambda)=\sum_{k=1}^{2} D_{j k}^{ \pm}(\rho) y_{k}(x, \rho), \quad x \in J_{ \pm},
$$

where

$$
D_{j k}^{+}(\rho)=\sum_{s=1}^{2} a_{s j} d_{s k}^{+}(\rho), \quad B_{k j}^{+}(\rho)=\frac{(-1)^{j-1}}{\operatorname{det} A} \sum_{s=1}^{2} a_{3-s, 3-j} b_{k s}^{+}(\rho),
$$

and $D_{j k}^{-}(\rho), B_{k j}^{-}(\rho)$ are defined by (2.15) and (2.17). Clearly,

$$
\left[D_{j k}^{ \pm}(\rho)\right]_{j, k=1,2}=\left(\left[B_{k j}^{ \pm}(\rho)\right]_{k, j=1,2}\right)^{-1} .
$$

For definiteness, in the sequel we confine ourselves to the most important particular case when $a_{12}=0$. Substituting the asymptotics (2.11), (2.13), and (2.18) for $b_{k j}^{ \pm}(\rho)$ and $d_{j k}^{ \pm}(\rho)$ into (2.15), (2.17), and (2.25), we calculate, for $|\rho| \rightarrow \infty, \rho \in \bar{S}_{k_{0}}$,

$$
\begin{gathered}
B_{k j}^{+}(\rho)=b_{k j}\left(a_{j j}\right)^{-1} \rho^{\mu_{j}}[1], \quad D_{j k}^{+}(\rho)=d_{j k} a_{j j} \rho^{-\mu_{j}}[1], \\
B_{k j}^{-}(\rho)=b_{k j} \exp \left(-i \pi \mu_{j}\right) \rho^{\mu_{j}}[1], \quad D_{j k}^{-}(\rho)=d_{j k} \exp \left(i \pi \mu_{j}\right) \rho^{-\mu_{j}}[1] .
\end{gathered}
$$

\section{Properties of the spectrum and the Weyl function}

3.1. Suppose that

$$
a_{11} \exp (2 \pi i \nu)-a_{22} \neq 0 .
$$

We will call (3.1) the regularity condition for sewing. Below, in Section 4 we provide a counterexample showing the importance of the regularity condition (3.1) for studying the boundary value problem $L$.

We denote

$$
\begin{aligned}
& \varphi_{1}(x, \lambda)=\sigma_{2}^{\prime}(0, \lambda) \sigma_{1}(x, \lambda)-\sigma_{1}^{\prime}(0, \lambda) \sigma_{2}(x, \lambda), \\
& \varphi_{2}(x, \lambda)=\sigma_{1}(0, \lambda) \sigma_{2}(x, \lambda)-\sigma_{2}(0, \lambda) \sigma_{1}(x, \lambda) .
\end{aligned}
$$

The functions $\varphi_{j}(x, \lambda), j=1,2$, are solutions of the differential equation (1.1) for $x \in J_{ \pm}$, and satisfy the initial conditions

$$
\varphi_{j}^{(m-1)}(0, \lambda)=\delta_{j m}, \quad j, m=1,2
$$

$\left(\delta_{j m}\right.$ is the Kronecker delta). By virtue of (2.23),

$$
\operatorname{det}\left[\varphi_{j}^{(m-1)}(x, \lambda)\right]_{j, m=1,2} \equiv \begin{cases}1, & x<a, \\ \operatorname{det} A, & x>a .\end{cases}
$$


LemmA 3.1. For $|\rho(x-a)| \geq 1, m=0,1,|\rho| \rightarrow \infty$, the following asymptotic formulae are valid:

$$
\begin{aligned}
\varphi_{j}^{(m)}(x, \lambda)= & \frac{1}{2}\left((-i \rho)^{m-j+1} \exp (-i \rho x)[1]_{a}+(i \rho)^{m-j+1} \exp (i \rho x)[1]_{a}\right), \quad x \in J_{-}, \\
\varphi_{j}^{(m)}(x, \lambda)= & \frac{1}{2}\left((-i \rho)^{m-j+1}\left(\xi_{12} \exp (-i \rho x)[1]_{a}+(-1)^{j-1} \xi_{22} \exp (-i \rho(x-2 a))[1]_{a}\right)\right. \\
& \left.+(i \rho)^{m-j+1}\left(\xi_{21} \exp (i \rho x)[1]_{a}+(-1)^{j-1} \xi_{11} \exp (i \rho(x-2 a))[1]_{a}\right)\right), \quad x \in J_{+},
\end{aligned}
$$

where

$$
\begin{aligned}
& {\left[\begin{array}{ll}
\xi_{11} & \xi_{12} \\
\xi_{21} & \xi_{22}
\end{array}\right]} \\
& \quad=\frac{1}{2 \sin \pi \nu}\left[\begin{array}{cc}
-a_{11} \exp (2 \pi i \nu)+a_{22} \exp (-2 \pi i \nu) & -i\left(a_{11} \exp (\pi i \nu)-a_{22} \exp (-\pi i v)\right) \\
-i\left(a_{11} \exp (\pi i v)-a_{22} \exp (-\pi i v)\right) & a_{11}-a_{22}
\end{array}\right] .
\end{aligned}
$$

We note that it follows from (3.1) that $\xi_{12} \neq 0, \xi_{21} \neq 0$.

Proof. Applying the fundamental system of solutions $\left\{y_{k}(x, \rho)\right\}_{k=1,2}$, we expand $\varphi_{j}(x, \lambda)$ for $x \in J_{+}$and $x \in J_{-}$separately:

$$
\varphi_{j}(x, \lambda)=\sum_{k=1}^{2} A_{j k}^{ \pm}(\rho) y_{k}(x, \rho), \quad x \in J_{ \pm} .
$$

For definiteness, let $\rho \in \bar{S}_{0}$ (other $\rho$ can be treated similarly). Then, $R_{k}=(-1)^{k-1} i$. First we calculate $A_{j k}^{-}(\rho)$ using the initial conditions (3.3):

$$
\sum_{k=1}^{2} A_{j k}^{-}(\rho) y_{k}^{(m-1)}(0, \rho)=\delta_{j m}
$$

Taking (2.20) into account, we get

$$
A_{j k}^{-}(\rho)=\frac{1}{2}\left((-1)^{k} i \rho\right)^{1-j} \exp \left((-1)^{k} i \rho a\right)[1]
$$

Together with (3.8) and (2.20), this yields (3.5).

In order to calculate $A_{j k}^{+}(\rho)$, we use sewing (matching) solutions with the help of the fundamental system of solutions $\left\{\sigma_{j}(x, \lambda)\right\}$ (see (3.2)). Substituting (2.24) into (3.8), we get

$$
\varphi_{j}(x, \lambda)=\sum_{k=1}^{2} A_{j k}^{ \pm}(\rho) y_{k}(x, \rho)=\sum_{s=1}^{2} \sigma_{s}(x, \lambda) \sum_{k=1}^{2} A_{j k}^{ \pm}(\rho) B_{k s}^{ \pm}(\rho), \quad x \in J_{ \pm},
$$

and hence,

$$
\sum_{k=1}^{2} A_{j k}^{+}(\rho) B_{k s}^{+}(\rho)=\sum_{k=1}^{2} A_{j k}^{-}(\rho) B_{k s}^{-}(\rho)
$$


172 Spectral analysis for differential operators with singularities

Since $\left(\left[B_{k j}^{ \pm}(\rho)\right]_{k, j=1,2}\right)^{-1}=\left[D_{j k}^{ \pm}(\rho)\right]_{j, k=1,2}$, the last equality yields

$$
A_{j k}^{ \pm}(\rho)=\sum_{s=1}^{2} A_{j s}^{\mp}(\rho) \xi_{s k}^{ \pm}(\rho)
$$

where

$$
\xi_{s k}^{ \pm}(\rho)=\sum_{m=1}^{2} B_{s m}^{\mp}(\rho) D_{m k}^{ \pm}(\rho)
$$

It follows from (3.14) and (2.27) that

$$
\xi_{s k}^{+}(\rho)=\sum_{m=1}^{2} b_{s m} d_{m k} a_{m m} \exp \left(-i \pi \mu_{m}\right)+O\left(\frac{1}{\rho}\right) .
$$

It was shown in [21] that

$$
b_{2 j}=b_{1 j} \exp \left(i \pi \mu_{j}\right), \quad b_{11} b_{12}=\frac{i}{\sin \pi \nu},
$$

and consequently,

$$
d_{j 1}=d_{j} \exp \left(-i \pi \mu_{j}\right), \quad d_{j 2}=d_{j}, \quad d_{1} d_{2}=-\frac{1}{4 i \sin \pi \nu},
$$

where $d_{1}=b_{12} / 2 i, d_{2}=-b_{11} / 2 i$. Together with (3.15), this yields

$$
\xi_{s k}^{+}(\rho)=\xi_{s k}+O\left(\frac{1}{\rho}\right)
$$

where the numbers $\xi_{s k}$ are defined by (3.7). Substituting (3.18) and (3.10) into (3.13), we calculate

$$
A_{j k}^{+}(\rho)=\frac{1}{2} \sum_{s=1}^{2}\left((-1)^{s} i \rho\right)^{1-j} \exp \left((-1)^{s} i \rho a\right)\left(\xi_{s k}+O\left(\frac{1}{\rho}\right)\right),
$$

hence, (3.8) and (2.14) yield (3.6). Lemma 3.1 is proved.

We denote

$$
\begin{gathered}
\varphi(x, \lambda)=\varphi_{1}(x, \lambda)+h \varphi_{2}(x, \lambda), \\
\Delta(\lambda)=V(\varphi)=\varphi^{\prime}(T, \lambda)+H \varphi(T, \lambda) .
\end{gathered}
$$

The function $\Delta(\lambda)$ is entire in $\lambda$, and its zeros $\left\{\lambda_{n}\right\}_{n \geq 0}$ coincide with the eigenvalues of the boundary value problem $L$. Moreover, if $\lambda_{n}$ is a zero of $\Delta(\lambda)$ of multiplicity $\kappa_{n}$, then the functions $\varphi_{n s}(x)=\left(\partial^{s} / \partial \lambda^{s}\right) \varphi(x, \lambda)_{\mid \lambda=\lambda_{n}}, s=\overline{0, \kappa_{n}-1}$ form the chain of e.a.f. for the eigenvalue $\lambda_{n}$. The function $\Delta(\lambda)$ is called the characteristic function for $L$. In view of 
(3.6), (3.20), and (3.21), we have

$$
\Delta(\lambda)=\Delta_{0}(\rho)\left(1+O\left(\frac{1}{\rho}\right)\right)
$$

where

$$
\begin{gathered}
\Delta_{0}(\rho)=\frac{i \rho}{2}\left(\xi_{21} \exp (i \rho T)-\xi_{12} \exp (-i \rho T)+\xi_{11} \exp (i \rho(T-2 a))\right. \\
\left.-\xi_{22} \exp (-i \rho(T-2 a))\right) .
\end{gathered}
$$

Using (3.22) and (3.23), by the well-known methods (see, e.g., [3]), one can obtain the following properties of the characteristic function $\Delta(\lambda)$ and the eigenvalues $\left\{\lambda_{n}\right\}_{n \geq 0}$ of the boundary value problem $L$ :

(1) for $|\rho| \rightarrow \infty, \Delta(\lambda)=O(|\rho| \exp (|\tau| T))$ where $\tau:=\operatorname{Im} \rho$;

(2) there exist $h>0, C_{h}>0$, such that

$$
|\Delta(\lambda)| \geq C_{h}|\rho| \exp (|\tau| T)
$$

for $|\tau| \geq h$. Hence, the eigenvalues $\lambda_{n}=\rho_{n}^{2}$ lie in the domain $|\operatorname{Im} \rho|<h$;

(3) the number $N_{\xi}$ of zeros of $\Delta(\lambda)$ in the rectangle $\Pi_{\xi}=\{\rho:|\operatorname{Im} \rho| \leq h, \operatorname{Re} \rho \in$ $[\xi, \xi+1]\}$ is bounded with respect to $\xi$;

(4) denote $G_{\delta}=\left\{\rho:\left|\rho-\rho_{n}\right| \geq \delta\right\}$. Then,

$$
|\Delta(\lambda)| \geq C_{\delta}|\rho| \exp (|\tau| T), \quad \rho \in G_{\delta} ;
$$

(5) there exist numbers $r_{N} \rightarrow \infty$ such that for sufficiently small $\delta>0$, the circles $|\rho|=$ $r_{N}$ lie in $G_{\delta}$ for all $N$;

(6) let $\left\{\rho_{n}^{0}\right\}$ be zeros of the function $\Delta_{0}(\rho)$ of the form (3.23). Then for $n \rightarrow \infty$,

$$
\rho_{n}=\rho_{n}^{0}+O\left(\frac{1}{\rho_{n}^{0}}\right) .
$$

3.2. We introduce the function

$$
M(\lambda)=\frac{\delta(\lambda)}{\Delta(\lambda)}
$$

where $\delta(\lambda):=V\left(\varphi_{2}\right)=\varphi_{2}^{\prime}(T, \lambda)+H \varphi_{2}(T, \lambda)$. Then the function

$$
\Phi(x, \lambda):=\varphi_{2}(x, \lambda)+M(\lambda) \varphi(x, \lambda)
$$

satisfies the differential equation (1.1), for $x \in J_{ \pm}$, and the boundary conditions $U(\Phi)=$ $1, V(\Phi)=0$. The functions $\Phi(x, \lambda)$ and $M(\lambda)$ are meromorphic in $\lambda$ with poles on the spectrum of the boundary value problem $L$. We will call $M(\lambda)$ the Weyl function since it is a generalization of the concept of the Weyl function for the classical Sturm-Liouville operators (when $\nu=1 / 2, A=E$ ). Clearly,

$$
\Phi(x, \lambda)=\frac{\psi(x, \lambda)}{\Delta(\lambda)},
$$


174 Spectral analysis for differential operators with singularities

where $\psi(x, \lambda)=V(\varphi) \varphi_{2}(x, \lambda)-V\left(\varphi_{2}\right) \varphi(x, \lambda)$. The function $\psi(x, \lambda)$ is entire in $\lambda$ and satisfies the differential equation (1.1) and the initial conditions

$$
\psi(T, \lambda)=-\operatorname{det} A, \quad \psi^{\prime}(T, \lambda)=H \operatorname{det} A .
$$

We note that by virtue of (3.4), (3.20), and (3.28),

$$
\langle\varphi(x, \lambda), \Phi(x, \lambda)\rangle \equiv \begin{cases}1, & x<a \\ \operatorname{det} A, & x>a\end{cases}
$$

where $\langle y, z\rangle:=y z^{\prime}-y^{\prime} z$.

Lemma 3.2. For $|\rho(x-a)| \geq 1, m=0,1,|\rho| \rightarrow \infty$, the following asymptotic formulae are valid:

$$
\begin{aligned}
& \psi^{(m)}(x, \lambda) \\
& =-\frac{1}{2} \operatorname{det} A\left((i \rho)^{m} \exp (-i \rho(T-x))[1]_{a}+(-i \rho)^{m} \exp (i \rho(T-x))[1]_{a}\right), \quad x \in J_{+}, \\
& \begin{array}{l}
\psi^{(m)}(x, \lambda) \\
=-\frac{1}{2}\left((i \rho)^{m}\left(\xi_{12} \exp (-i \rho(T-a))-\xi_{11} \exp (i \rho(T-a))\right) \exp (-i \rho(a-x))[1]_{a}\right. \\
\quad+(-i \rho)^{m}\left(\xi_{21} \exp (i \rho(T-a))\right. \\
\left.\left.\quad-\xi_{22} \exp (-i \rho(T-a))\right) \exp (i \rho(a-x))[1]_{a}\right), \quad x \in J_{-} .
\end{array}
\end{aligned}
$$

Proof. Using the fundamental system of solutions $\left\{y_{k}(x, \rho)\right\}_{k=1,2}$, one can write

$$
\psi(x, \lambda)=\sum_{k=1}^{2} A_{k}^{ \pm}(\rho) y_{k}(x, \rho), \quad x \in J_{ \pm} .
$$

Taking (3.30) into account, we calculate

$$
A_{1}^{+}(\rho)=-\frac{\operatorname{det} A}{w(\rho)} V\left(y_{2}\right), \quad A_{2}^{+}(\rho)=\frac{\operatorname{det} A}{w(\rho)} V\left(y_{1}\right), \quad w(\rho)=\operatorname{det}\left[y_{k}^{(m-1)}(T, \rho)\right]_{k, m=1,2} .
$$

For definiteness, let $\rho \in \bar{S}_{0}$. Then $R_{k}=(-1)^{k-1} i$ and, by virtue of (2.19),

$$
A_{k}^{+}(\rho)=-\frac{1}{2} \operatorname{det} A \exp \left((-1)^{k} i \rho(T-a)\right)[1] .
$$

Substituting (3.36) and (2.19) into (3.34), we arrive at (3.32).

Furthermore, repeating the arguments in the proof of Lemma 3.1 we infer similarly to (3.13):

$$
A_{k}^{-}(\rho)=\sum_{s=1}^{2} A_{s}^{+}(\rho) \xi_{s k}^{-}(\rho)
$$


Since $\left[\xi_{s k}^{-}(\rho)\right]_{s, k=1,2}=\left(\left[\xi_{s k}^{+}(\rho)\right]_{s, k=1,2}\right)^{-1}$, it follows from (3.18), (3.36), and (3.37) that

$$
A_{k}^{-}(\rho)=\frac{(-1)^{k}}{2} \sum_{s=1}^{2} \xi_{3-k, j} \exp \left((-1)^{j-1} i \rho(T-a)\right)[1]_{a},
$$

and we arrive at (3.33).

Corollary 3.3. For $\rho \in G_{\delta}$,

$$
\begin{gathered}
\left|\Phi^{(m)}(x, \lambda)\right| \leq C_{\delta}|\rho|^{m-1} \exp (-|\tau| x), \quad|\rho(x-a)| \geq 1, \\
|M(\lambda)| \leq C_{\delta}|\rho|^{-1} .
\end{gathered}
$$

Indeed, (3.39) follows from (3.29), (3.25), and Lemma 3.2. By virtue of (3.28), $M(\lambda)=$ $\Phi(0, \lambda)$, and (3.40) follows from (3.39).

\section{The completeness theorem}

Let $\alpha$ be a real number, and let $1 \leq p<\infty$. We consider the Banach spaces $B_{\alpha, p}=\{f(x)$ : $\left.f(x) x^{-\alpha} \in \mathscr{L}_{p}(0, T)\right\}$ with the norm $\|f\|_{\alpha, p}=\left\|f(x) x^{-\alpha}\right\|_{p}$, where $\|\cdot\|_{p}$ is the norm in the space $\mathscr{L}_{p}(0, T)$. We denote by $B_{\alpha, p}^{*}$ the dual space. Clearly, $B_{\alpha, p}^{*}=B_{-\alpha, q}\left(p^{-1}+q^{-1}=1\right.$, $p>1)$. We show that

$$
B_{\alpha, p} \subseteq B_{\beta, s}, \quad 1 \leq s \leq p<\infty, \beta-\alpha<s^{-1}-p^{-1}
$$

(here the symbol $\subseteq$ denotes dense embedding [12, page 9]).

Indeed, for $\alpha \geq \beta, s \leq p$, we have $B_{\alpha, p} \subseteq B_{\beta, p}, B_{\beta, p} \subseteq B_{\beta, s}$, and consequently (4.1) is obvious. Assume now that $\alpha<\beta, s<p$. We consider the function $f(x) \in B_{\alpha, p}$. Let $r=p / s$, $r^{\prime}=p /(p-s)$. Then, $r^{-1}+\left(r^{\prime}\right)^{-1}=1$. Since $\beta-\alpha<s^{-1}<p^{-1}$, we have $(\alpha-\beta) s r^{\prime}>-1$. Applying Hölder's inequality, we obtain $\left\|f(x) x^{-\beta}\right\|_{s} \leq\left\|f(x) x^{-\alpha}\right\|_{s r}\left\|x^{\alpha-\beta}\right\|_{s r^{\prime}}$, and consequently $\|f\|_{\beta, s} \leq C\|f\|_{\alpha, p}$. Since $B_{\alpha, p}$ is dence in $B_{\beta, s}$, it follows that (4.1) holds. In particular, it follows from (4.1) that $B_{\alpha, p} \subseteq \mathscr{L}_{s}$ for $1 \leq s \leq p<\infty, \alpha>p^{-1}-s^{-1}$.

We set $\omega=-\operatorname{Re} \nu+1 / 2$.

THEOREM 4.1. The system of e.a.f. of the boundary value problem $L$ is complete in the space $B_{\beta, s}$ for $1 \leq s<\infty, \beta<\omega+1 / s$.

Proof. Let $\left\{\varphi_{\ell}(x)\right\}$ be e.a.f. of $L$, and let a function $f(x)$ be such that

$$
f(x)(x-a)^{\omega} \in \mathscr{L}(0, T), \quad \int_{0}^{T} f(x) \varphi_{\ell}(x) d x=0 .
$$

We consider the function

$$
Y(x, \lambda)=\Phi^{*}(x, \lambda) \int_{0}^{x} f(t) \varphi(t, \lambda) d t+\varphi^{*}(x, \lambda) \int_{x}^{T} f(t) \Phi(t, \lambda) d t,
$$

where

$$
\Phi^{*}(x, \lambda)=\frac{\Phi(x, \lambda)}{\eta(x)}, \quad \varphi^{*}(x, \lambda)=\frac{\varphi(x, \lambda)}{\eta(x)}, \quad \eta(x)= \begin{cases}1, & x<a \\ \operatorname{det} A, & x>a\end{cases}
$$


176 Spectral analysis for differential operators with singularities

In view of (3.31), a direct computation yields

$$
\ell Y-\lambda Y=-f(x), \quad x \in J_{ \pm}
$$

We now estimate the function $Y(x, \lambda)$ for $\rho \in G_{\delta}$. For this, we will use (3.39) and the estimate

$$
\left|\varphi^{(m)}(x, \lambda)\right| \leq C|\rho|^{m} \exp (|\tau| x), \quad|\rho(x-a)| \geq 1, m=0,1,
$$

which follows from (3.20) and Lemma 3.1.

Fix $x \in[0, T] \backslash\{a\}$. Then, $|\rho(x-a)| \geq 1$ for sufficiently large $\rho$. Let $x \in J_{+}$. We have

$$
\begin{aligned}
|Y(x, \lambda)| \leq & C_{\delta}|\rho|^{-1} \exp (-|\tau| x)\left(\int_{0}^{a-|\rho|^{-1}}+\int_{a-|\rho|^{-1}}^{a+|\rho|^{-1}}+\int_{a+|\rho|^{-1}}^{x}\right)|f(t) \varphi(t, \lambda)| d t \\
& +C \exp (|\tau| x) \int_{x}^{T}|f(t) \Phi(t, \lambda)| d t
\end{aligned}
$$

and consequently,

$$
|Y(x, \lambda)| \leq C_{\delta}\left(\frac{1}{|\rho|} \exp (-|\tau| x) \int_{\gamma_{\rho}}|f(t) \varphi(t, \lambda)| d t+\frac{1}{|\rho|} \int_{\Gamma_{\rho}}|f(t)| d t\right)
$$

where $|\rho(x-a)| \geq 1, x \in J_{+}, \rho \in G_{\delta}, \gamma_{\rho}=\{t:|\rho(t-a)| \leq 1\}$, and $\Gamma_{\rho}=\{t:|\rho(t-a)| \geq 1\}$. Analogously, for $|\rho(x-a)| \geq 1, x \in J_{-}, \rho \in G_{\delta}$,

$$
|Y(x, \lambda)| \leq C_{\delta}\left(\exp (|\tau| x) \int_{\gamma_{\rho}}|f(t) \Phi(t, \lambda)| d t+\frac{1}{|\rho|} \int_{\Gamma_{\rho}}|f(t)| d t\right) .
$$

In order to estimate the integrals on $\gamma_{\rho}$ in (4.8) and (4.9), we need the following assertion.

Lemma 4.2. For $|\rho(x-a)| \leq 1$,

$$
\begin{gathered}
|\varphi(x, \lambda)| \leq C|\rho(x-a)|^{\omega} \exp (|\tau| a), \\
|\Phi(x, \lambda)| \leq C_{\delta}|\rho|^{-1}|\rho(x-a)|^{\omega} \exp (-|\tau| a), \quad \rho \in G_{\delta} .
\end{gathered}
$$

Proof. It follows from (3.2), (3.20), and (3.30) that

$$
\begin{aligned}
& \varphi(x, \lambda)=U\left(\sigma_{2}\right) \sigma_{1}(x, \lambda)-U\left(\sigma_{1}\right) \sigma_{2}(x, \lambda), \quad x \in J_{ \pm}, \\
& \psi(x, \lambda)=\operatorname{det} A\left(V\left(\sigma_{1}\right) \sigma_{2}(x, \lambda)-V\left(\sigma_{2}\right) \sigma_{1}(x, \lambda)\right), \quad x \in J_{ \pm} .
\end{aligned}
$$


By virtue of (2.24), (2.27), (2.19), and (2.20),

$$
\begin{aligned}
\left|\sigma_{j}^{(m)}(0, \lambda)\right| \leq C|\rho|^{m-\operatorname{Re} \mu_{j}} \exp (|\tau| a), \\
\left|\sigma_{j}^{(m)}(T, \lambda)\right| \leq C|\rho|^{m-\operatorname{Re} \mu_{j}} \exp (|\tau|(T-a)),
\end{aligned}
$$

and consequently,

$$
\begin{aligned}
& \left|U\left(\sigma_{j}\right)\right| \leq C|\rho|^{1-\operatorname{Re} \mu_{j}} \exp (|\tau| a), \\
& \left|V\left(\sigma_{j}\right)\right| \leq C|\rho|^{1-\operatorname{Re} \mu_{j}} \exp (|\tau|(T-a)) .
\end{aligned}
$$

Since $a_{12}=0$, it follows from (2.6) and (2.22) that

$$
\left|\sigma_{j}(x, \lambda)\right| \leq C\left|(x-a)^{\mu_{j}}\right|, \quad|\rho(x-a)| \geq 1, \quad x \in J_{ \pm} .
$$

Using (4.11), (4.12), (4.13), (4.14), (3.25), and (3.29), we arrive at (4.10).

We return to the proof of Theorem 4.1. Substituting (4.10) into (4.8) and (4.9), we get

$$
\begin{gathered}
|Y(x, \lambda)| \leq \frac{C_{\delta}}{|\rho|}\left(\int_{\gamma_{\rho}}|\rho(t-a)|^{\omega}|f(t)| d t+\int_{\Gamma_{\rho}}|f(t)| d t\right), \\
|\rho(x-a)| \geq 1, \quad \rho \in G_{\delta} .
\end{gathered}
$$

If $\operatorname{Re} v \geq 1 / 2$, then $\omega \leq 0$, and

$$
|Y(x, \lambda)| \leq \frac{C_{\delta}}{|\rho|}, \quad|\rho(x-a)| \geq 1, \quad \rho \in G_{\delta} .
$$

If $\operatorname{Re} v<1 / 2$, then $\omega>0$, and

$$
|Y(x, \lambda)| \leq \frac{C_{\delta}}{|\rho|^{\operatorname{Re} \nu+1 / 2}}, \quad|\rho(x-a)| \geq 1, \quad \rho \in G_{\delta} .
$$

Thus, we conclude that for each fixed $x \in J_{ \pm}$,

$$
Y(x, \lambda)=o(1), \quad|\rho| \longrightarrow \infty, \quad \rho \in G_{\delta}
$$

Furthermore, taking (3.28) into account, one can rewrite $Y(x, \lambda)$ as follows

$$
Y(x, \lambda)=M(\lambda) \varphi^{*}(x, \lambda) \int_{0}^{T} f(t) \varphi(t, \lambda) d t+Y_{0}(x, \lambda)
$$

where the function $Y_{0}(x, \lambda)$ is entire in $\lambda$ for each fixed $x \neq a$. By virtue of (4.2), the last relation implies that $y(x, \lambda)$ is entire in $\lambda$ for each fixed $x \neq a$. Together with (4.18), this yields $Y(x, \lambda) \equiv 0$. Using now (4.5), we conclude that $f(x)=0$ a.e. on $(0, T)$. 
Thus, we have proved that for each $p(1 \leq p<\infty)$, the system of e.a.f. of $L$ is complete in $B_{\omega, p}$. Since $\beta-\omega<1 / s$, we have $\beta-\omega<1 / s-1 / p$ for sufficiently large $p$, and according to (4.1), $B_{\omega, p} \subseteq B_{\beta, s}$. Consequently, the system of e.a.f. of $L$ is complete in $B_{\beta, s}$ for $1 \leq s<$ $\infty, \beta<\omega+1 / s$. Theorem 4.1 is proved.

Corollary 4.3. The system of e.a.f. of $L$ is complete in $\mathscr{L}_{s}(0, T)$ for $1 / s>\operatorname{Re} v-1 / 2$.

Remark 4.4. We provide here a counterexample showing the importance of the regularity condition (3.1). We consider the boundary value problem $L$ with $\nu_{0}=0, q(x) \equiv 0, T=\pi$, $h=H=0, a=3 \pi / 4, a_{11}=-a_{22}=1, a_{21}=a_{12}=0$, that is, we consider the boundary value problem

$$
\begin{gathered}
-y^{\prime \prime}=\lambda y, \quad 0<x<\pi, \\
y^{\prime}(0)=y^{\prime}(\pi)=0, \\
y^{(m)}(a+0)=(-1)^{m} y^{(m)}(a-0), \quad m=0,1, a=\frac{3 \pi}{4} .
\end{gathered}
$$

For this problem, the regularity condition (3.1) is not fulfilled. The characteristic function for (4.20) has the form

$$
\Delta(\lambda)=\rho \sin \rho(2 a-T)
$$

The eigenvalues $\lambda_{n}=\rho_{n}^{2}$ are $\rho_{n}=2 n, n \geq 0$, and the eigenfunctions have the form

$$
y_{n}(x)= \begin{cases}\cos 2 n x, & x \leq \frac{3 \pi}{4}, \\ (-1)^{n} \cos 2 n x, & x>\frac{3 \pi}{4} .\end{cases}
$$

The system of functions $\left\{y_{n}(x)\right\}_{n \geq 0}$ is not complete in $B_{\beta, s}$ for $1 \leq s<\infty, \beta<1+1 / s$.

\section{Inverse problems}

In this section, we study the inverse problem of recovering the boundary value problem $L$ from its spectral characteristics. We consider two formulations of the inverse problems: to construct $L$ from the Weyl function and from discrete spectral characteristics. These inverse problems are the generalization of the well-known inverse problems for the classical Sturm-Liouville operators (see [16]).

We formulate the uniqueness theorem for the solution of the inverse problem of recovering $L$ from the Weyl function $M(\lambda)$. For this, we agree that together with $L$, we consider a boundary value problem $\tilde{L}$ of the same form but with different coefficients $\tilde{q}(x), \tilde{v}_{0}$, $\tilde{h}$, and $\tilde{H}$. If a certain symbol $\alpha$ denotes an object related to $L$, then $\tilde{\alpha}$ will denote the analogous object related to $\tilde{L}$.

Theorem 5.1. If $M(\lambda)=\tilde{M}(\lambda)$, then $L=\tilde{L}$. Thus, the specification of the Weyl function uniquely determines the boundary value problem.

Proof. Consider the functions

$$
P_{m}(x, \lambda)=\varphi(x, \lambda) \tilde{\Phi}^{(m)}(x, \lambda)-\Phi(x, \lambda) \tilde{\varphi}^{(m)}(x, \lambda), \quad m=0,1 .
$$


For each fixed $x \in[0, T] \backslash\{a\}$, the functions $P_{m}(x, \lambda)$ are meromorphic in $\lambda$ with poles at $\lambda=\lambda_{n}$ and $\lambda=\tilde{\lambda}_{n}$. Fix $x \in[0, T] \backslash\{a\}$. Then $|\rho(x-a)| \geq 1$ for sufficiently large $\rho$. Denote $G_{\delta}^{0}=G_{\delta} \cap \tilde{G}_{\delta}$. By virtue of (3.39) and (4.6),

$$
\left|P_{m}(x, \lambda)\right| \leq C_{\delta}|\rho|^{m-1}, \quad \rho \in G_{\delta}, \quad|\rho(x-a)| \geq 1 .
$$

Substituting (3.28) into (5.1), we obtain

$$
\begin{aligned}
P_{m}(x, \lambda)= & \left(\varphi(x, \lambda) \tilde{\varphi}_{2}^{(m)}(x, \lambda)-\varphi_{2}(x, \lambda) \tilde{\varphi}^{(m)}(x, \lambda)\right) \\
& +(\tilde{M}(\lambda)-M(\lambda)) \varphi(x, \lambda) \tilde{\varphi}^{(m)}(x, \lambda) .
\end{aligned}
$$

Since $\tilde{M}(\lambda)=M(\lambda)$, the last relation implies that for each fixed $x \in[0, T] \backslash\{a\}$, the functions $P_{m}(x, \lambda)$ are entire in $\lambda$. Together with (5.2), this yields

$$
P_{0}(x, \lambda) \equiv 0, \quad P_{1}(x, \lambda) \equiv P(x) .
$$

But then, according to (5.1),

$$
\begin{aligned}
\varphi(x, \lambda) \tilde{\Phi}(x, \lambda) & =\Phi(x, \lambda) \tilde{\varphi}(x, \lambda), \\
P(x) \tilde{\varphi}(x, \lambda) & =\left(\varphi(x, \lambda) \tilde{\Phi}^{\prime}(x, \lambda)-\Phi(x, \lambda) \tilde{\varphi}^{\prime}(x, \lambda)\right) \tilde{\varphi}(x, \lambda) \\
& =\left(\tilde{\varphi}(x, \lambda) \tilde{\Phi}^{\prime}(x, \lambda)-\tilde{\Phi}(x, \lambda) \tilde{\varphi}^{\prime}(x, \lambda)\right) \varphi(x, \lambda) \\
& =\tilde{\eta}(x) \varphi(x, \lambda) .
\end{aligned}
$$

Consequently,

$$
\frac{\varphi(x, \lambda)}{\tilde{\varphi}(x, \lambda)}=\frac{\Phi(x, \lambda)}{\tilde{\Phi}(x, \lambda)}=\frac{P(x)}{\tilde{\eta}(x)} .
$$

Furthermore, it follows from (3.5), (3.6), and (3.20) that for $|\rho| \rightarrow \infty, \arg \rho \in[\varepsilon, \pi-\varepsilon]$, $\varepsilon>0$,

$$
\begin{aligned}
\varphi(x, \lambda) & =\frac{1}{2} \exp (-i \rho x)[1]_{a}, \quad x<a \\
\varphi(x, \lambda) & =\frac{1}{2} \xi_{12} \exp (-i \rho x)[1]_{a}, \quad x>a .
\end{aligned}
$$

Similarly, by virtue of (3.32), (3.33), (3.29) and (3.23),

$$
\begin{aligned}
& \Phi(x, \lambda)=(i \rho)^{-1} \exp (i \rho x)[1]_{a}, \quad x<a, \\
& \Phi(x, \lambda)=\operatorname{det} A\left(i \rho \xi_{12}\right)^{-1} \exp (i \rho x)[1]_{a}, \quad x>a,
\end{aligned}
$$

for $|\rho| \rightarrow \infty, \arg \rho \in[\varepsilon, \pi-\varepsilon], \varepsilon>0$. Substituting (5.7) and (5.8) into (5.6), we get $P(x) \equiv$ 1 for $x<a$, and $P(x) \equiv P_{*}-$ const for $x>a$, that is, $\varphi(x, \lambda) \equiv \tilde{\varphi}(x, \lambda), \Phi(x, \lambda) \equiv \tilde{\Phi}(x, \lambda)$ for $x<a$, and $\varphi(x, \lambda) \equiv C \tilde{\varphi}(x, \lambda), \Phi(x, \lambda) \equiv C \tilde{\Phi}(x, \lambda)$ for $x>a$. Consequently, $\nu_{0}=\tilde{\nu}_{0}, q(x)=$ $\tilde{q}(x)$ a.e. on $(0, T), h=\tilde{h}$, and $H=\tilde{H}$. Theorem 5.1 is proved. 
Now we consider the inverse problem of recovering $L$ from discrete spectral characteristics. For brevity, we confine ourselves to the case when all zeros of $\Delta(\lambda)$ are simple. We denote

$$
\alpha_{n}=\underset{\lambda=\lambda_{n}}{\operatorname{Res}} M(\lambda)
$$

The numbers $\left\{\lambda_{n}, \alpha_{n}\right\}_{n \geq 0}$ are called the spectral data of $L$.

Theorem 5.2. If $\lambda_{n}=\tilde{\lambda}_{n}, \alpha_{n}=\tilde{\alpha}_{n}$ for all $n \geq 0$, then $L=\tilde{L}$.

Proof. Let $\Gamma=\left\{\lambda=u+i v: u=\left(2 h^{2}\right)^{-2} v^{2}-h^{2}\right\}$ be the image of $\operatorname{Im} \rho= \pm h$ under the mapping $\lambda=\rho^{2}$. Denote $\Gamma_{N}=\Gamma \cap\left\{\lambda:|\lambda| \leq r_{N}\right\}$, and consider the closed contours $\Gamma_{N 0}=$ $\Gamma_{N} \cup\left\{\lambda:|\lambda|=r_{N}, \lambda \notin \operatorname{int} \Gamma\right\}, \Gamma_{N 1}=\Gamma_{N} \cup\left\{\lambda:|\lambda|=r_{N}, \lambda \in \operatorname{int} \Gamma\right\}$ (with counterclockwise circuit). Since the Weyl function $M(\lambda)$ is regular for $\lambda \in \operatorname{int} \Gamma_{N 0}$, we get by Cauchy's integral formula that

$$
M(\lambda)=\frac{1}{2 \pi i} \int_{\Gamma_{N 0}} \frac{M(\mu)}{\mu-\lambda} d \mu, \quad \lambda \in \operatorname{int} \Gamma_{N 0}
$$

By virtue of (3.40),

$$
\lim _{N \rightarrow \infty} \frac{1}{2 \pi i} \int_{|\mu|=r_{N}} \frac{M(\mu)}{\mu-\lambda} d \mu=0,
$$

and consequently,

$$
M(\lambda)=\lim _{N \rightarrow \infty} \frac{1}{2 \pi i} \int_{\Gamma_{N 1}} \frac{M(\mu)}{\lambda-\mu} d \mu .
$$

Calculating this integral by the residue theorem, we arrive at

$$
M(\lambda)=\sum_{n=0}^{\infty} \frac{\alpha_{n}}{\lambda-\lambda_{n}},
$$

where the series converge "with brackets":

$$
\sum_{n=0}^{\infty}=\lim _{N \rightarrow \infty} \sum_{\left|\lambda_{n}\right|<r_{N}^{2}} .
$$

Under the assumptions of Theorem 5.2, it follows from (5.13) that $M(\lambda)=\tilde{M}(\lambda)$. Applying Theorem 5.1, we get $L=\tilde{L}$.

Remark 5.3. Using the obtained results and the method of spectral mappings [27], one can obtain an algorithm for the solution of the inverse problems considered, along with necessary and sufficient conditions of their solvability (in analogous manner as in [25]).

\section{Acknowledgment}

This research was supported in part by Grants E02-1.0-186 and UR.04.01.042 of the Education Ministry of the Russian Federation. 


\section{References}

[1] T. Aktosun, M. Klaus, and C. van der Mee, Recovery of discontinuities in a non-homogeneous medium, Inverse Problems 12 (1996), no. 1, 1-25.

[2] R. S. Anderssen, The effect of discontinuities in density and shear velocity on the asymptotic overtone structure of tortional eigenfrequencies of the Earth, Earth Geophys. J.R. astr. Soc. $\mathbf{5 0}$ (1997), 303-309.

[3] R. Bellman and K. L. Cooke, Differential-Difference Equations, Academic Press, New York, 1963.

[4] R. Carlson, Inverse spectral theory for some singular Sturm-Liouville problems, J. Differential Equations 106 (1993), no. 1, 121-140.

[5] A. Constantin, On the inverse spectral problem for the Camassa-Holm equation, J. Funct. Anal. 155 (1998), no. 2, 352-363.

[6] W. Eberhard, G. Freiling, and A. Schneider, On the distribution of the eigenvalues of a class of indefinite eigenvalue problems, Differential Integral Equations 3 (1990), no. 6, 1167-1179.

[7] G. Freiling and V. A. Yurko, Reconstructing parameters of a medium from incomplete spectral information, Results Math. 35 (1999), no. 3-4, 228-249.

[8] M. G. Gasymov, Determination of a Sturm-Liouville equation with a singularity by two spectra, Dokl. Akad. Nauk SSSR 161 (1965), 274-276 (Russian).

[9] F. Gesztesy, A complete spectral characterization of the double commutation method, J. Funct. Anal. 117 (1993), no. 2, 401-446.

[10] O. H. Hald, Discontinuous inverse eigenvalue problems, Comm. Pure Appl. Math. 37 (1984), no. 5, 539-577.

[11] M. Kobayashi, A uniqueness proof for discontinuous inverse Sturm-Liouville problems with symmetric potentials, Inverse Problems 5 (1989), no. 5, 767-781.

[12] S. G. Kreĭn, Ju. I. Petunin, and E. M. Semënov, Interpolation of Linear Operators, Nauka, Moscow, 1978 (Russian), English translation, American Mathematical Society, Rhode Island, 1982.

[13] F. R. Lapwood and T. Usami, Free Oscillations of the Earth, Cambridge University Press, Cambridge, 1981.

[14] B. M. Levitan and I. S. Sargsjan, Introduction to Spectral Theory: Selfadjoint Ordinary Differential Operators, Translations of Mathematical Monographs, vol. 39, American Mathematical Society, Rhode Island, 1975.

[15] O. N. Litvinenko and V. I. Soshnikov, The Theory of Heterogenious Lines and Their Applications in Radio Engineering, Radio i Svyaz, Moscow, 1964 (Russian).

[16] V. A. Marchenko, Sturm-Liouville Operators and Their Applications, Izdat. Naukova Dumka, Kiev, 1977 (Russian), English translation, Birkhäuser, Basel, 1986.

[17] J. R. McLaughlin, Analytical methods for recovering coefficients in differential equations from spectral data, SIAM Rev. 28 (1986), no. 1, 53-72.

[18] V. P. Meschanov and A. L. Feldstein, Automatic Design of Directional Couplers, Radio i Svyaz, Moscow, 1980 (Russian).

[19] J. Pöschel and E. Trubowitz, Inverse Spectral Theory, Pure and Applied Mathematics, vol. 130, Academic Press, Massachusetts, 1987.

[20] V. V. Staševskaya, On inverse problems of spectral analysis for a class of differential equations, Dokl. Akad. Nauk SSSR 93 (1953), 409-411 (Russian).

[21] V. A. Yurko, Inverse problem for differential equations with a singularity, Differ. Uravn. 28 (1992), no. 8, 1355-1362 (Russian), translated in Differential Equations 28 (1992), no. 8, 11001107.

[22] On higher-order differential operators with a singular point, Inverse Problems 9 (1993), no. 4, 495-502. 
182 Spectral analysis for differential operators with singularities

[23] On higher-order differential operators with a regular singularity, Mat. Sb. 186 (1995), no. 6, 133-160 (Russian), translation in Sb. Math. 186 (1995), no. 6, 901-928.

[24] Integral transforms connected with differential operators having singularities inside the interval, Integral Transform. Spec. Funct. 5 (1997), no. 3-4, 309-322.

[25] - On the reconstruction of Sturm-Liouville differential operators with singularities inside the interval, Mat. Zametki 64 (1998), no. 1, 143-156 (Russian), translated in Math. Notes 64 (1998), no. 1-2, 121-132.

[26] Integral transforms connected with discontinuous boundary value problems, Integral Transform. Spec. Funct. 10 (2000), no. 2, 141-164.

[27] - Inverse Spectral Problems for Differential Operators and Their Applications, Analytical Methods and Special Functions, vol. 2, Gordon and Breach Science Publishers, Amsterdam, 2000.

[28] L. A. Zhornitskaya and V. S. Serov, Inverse eigenvalue problems for a singular Sturm-Liouville operator on [0, 1], Inverse Problems 10 (1994), no. 4, 975-987.

Vjacheslav Anatoljevich Yurko: Department of Mathematics, Saratov State University, Astrakhanskaya 83 , Saratov 410026 , Russia

E-mail address: yurkova@info.sgu.ru 


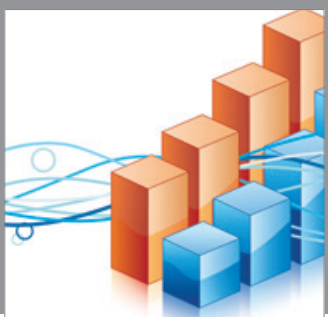

Advances in

Operations Research

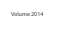

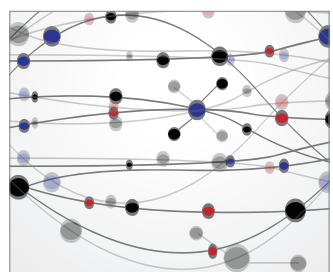

\section{The Scientific} World Journal


International Journal of

Mathematics and

Mathematical

Sciences


Journal of

Applied Mathematics
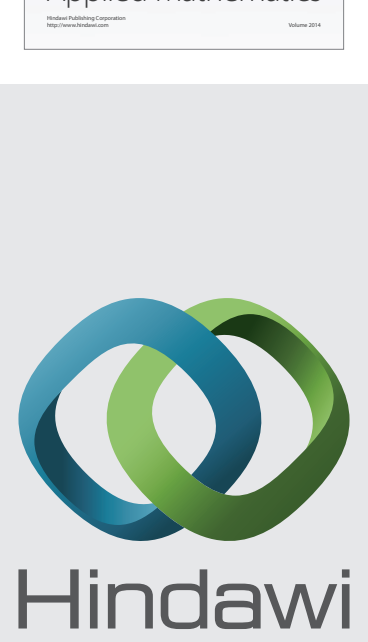

Submit your manuscripts at http://www.hindawi.com
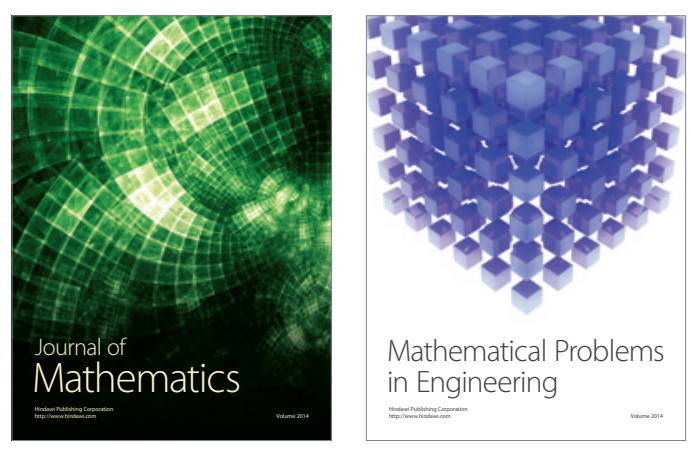

Mathematical Problems in Engineering
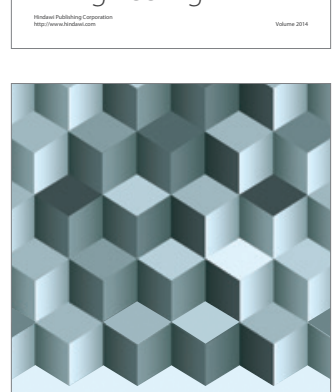

Journal of

Function Spaces
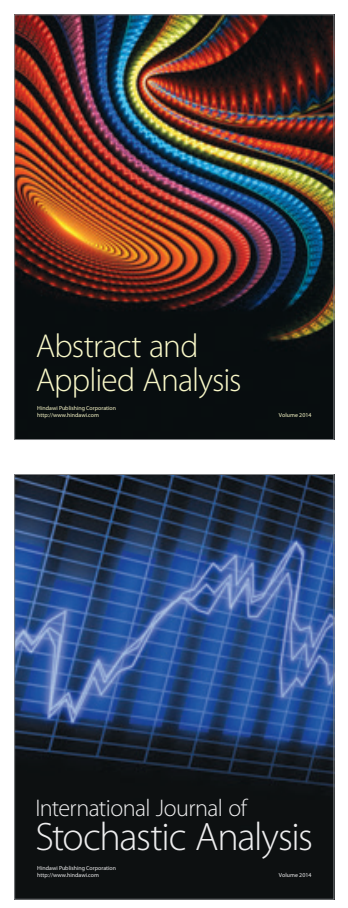

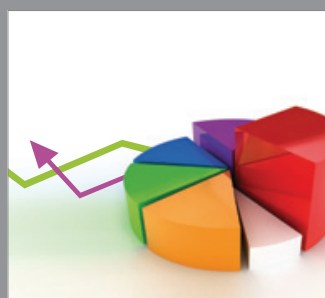

ournal of

Probability and Statistics

Promensencen
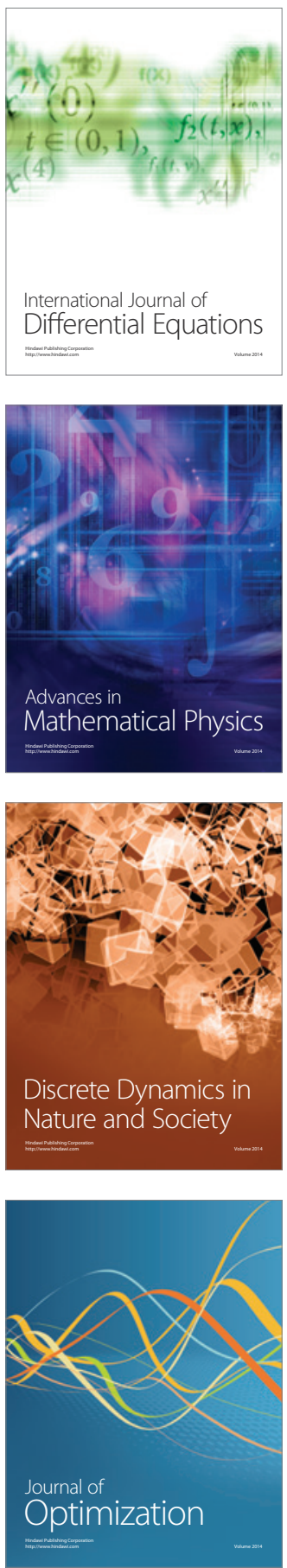